\title{
Appropriateness of computed tomography and magnetic resonance imaging scans in a rural regional hospital in South Africa: A 6-year follow-up study
}

\author{
P E Fouche, ${ }^{1}$ MB ChB, NDip ClinTech, BTech ClinTech, BSc Hons; L S Jenkins, ${ }^{2}$ MB ChB, MFamMed, Dip Obst (SA), DA (SA), DHSM, \\ FCFP (SA), PhD; A Vermeulen, ${ }^{3}$ MB BCh, FCP (SA), Cert Nephrology (SA), MMed (Int Med) \\ ${ }^{1}$ George Hospital, George, South Africa \\ ${ }^{2}$ Department of Family and Emergency Medicine, George Hospital, George; Department of Family and Emergency Medicine, Faculty of Medicine \\ and Health Sciences, Stellenbosch University, Cape Town; and Primary Health Care Directorate, Faculty of Health Sciences, \\ University of Cape Town, South Africa \\ ${ }^{3}$ Renal Unit, Department of Internal Medicine, George Hospital, George, South Africa
}

Corresponding author: L S Jenkins (louis.jenkins@westerncape.gov.za)

\begin{abstract}
Background. Requests for computed tomography (CT) and magnetic resonance imaging (MRI) scans by doctors with different levels of experience have cost and risk implications globally. Evidence-based appropriateness criteria guide doctors to the suitable use of radiology imaging. There are few studies regarding appropriateness of CT requests in the South African (SA) context. Previous research in the Garden Route district of Western Cape Province, SA, evaluated the appropriateness of scans.

Objectives. To review the appropriateness of CT and MRI scans done in a 6-year follow-up study at one facility.

Methods. This was a retrospective descriptive study. All CT and MRI scans performed during October 2018 at George Hospital were classified according to American College of Radiology guidelines as: usually appropriate (UA), might be appropriate (MBA), or not appropriate (NA). Stratified analysis allowed simple statistics and some comparison with the previous study.

Results. A total of 515 CT and MRI scans were included, of which $81.4 \%$ were UA, $7.4 \%$ MBA and $11.2 \%$ NA. Most scans were requested by medical officers $(n=255)$, followed by consultants $(n=126)$ and junior doctors $(n=70)$. Medical officers made the majority of inappropriate requests. Second-year interns requested the lowest number of inappropriate scans, with registrars not requesting any inappropriate scans. Most of the inappropriate scans were requested after hours. Thirty-seven of the $123(30.1 \%)$ after-hours scans were inappropriately requested compared with 21 of 392 (5.4\%) scans during normal working hours, which were inappropriate.

Conclusions. Although the majority of scans were being ordered appropriately, pre-authorisation by experienced physicians and incorporation of guidelines would make requests more complete and possibly more appropriate, especially after hours.
\end{abstract}

S Afr Med J 2021;111(1):46-51. https://doi.org/10.7196/SAMJ.2021.v11111.14860

Since its introduction in the 1970s, computed tomography (CT) and magnetic resonance imaging (MRI) have revolutionised diagnostic decision-making worldwide. ${ }^{[1]}$ The global CT market was worth USD6.6 billion in 2019 and is expected to reach USD8.2 billion by 2024. ${ }^{[2]}$ Medical imaging radiation contamination is now equivalent to background radiation levels in the USA. ${ }^{[3]}$ This is due to the speed of acquisition, ready availability and access to high-quality diagnostic information that favours the use of CT in current clinical practice. Radiographic examinations aid the physician's clinical diagnosis or guide treatment, as in the case of surgical planning. CT and MRI scans are requested by a variety of physicians with different levels of experience. It is generally believed that senior physicians use this valuable resource in a more insightful manner than junior physicians, whose diagnostic and professional uncertainty could contribute to inappropriate use of imaging to support clinical judgement. Ionising medical imaging use, such as CT scans, should ultimately be in the patients' best interests and improve health outcomes. However, increased use of CT and MRI scans has significant risks and implications, including increased costs to the patient or facility, longterm effects of radiation exposure to patients and staff, and increased workload to radiographers and radiologists. ${ }^{[4]}$

The appropriate use of MRI and CT scans has medical and economic implications, influenced by e.g. medical liability fears, patients' demands, regional differences in practice style and physician experience and training in newer imaging modalities. The American College of Radiology (ACR) developed an evidence-based set of appropriateness criteria (AC) intended to guide doctors to the appropriate use of radiology imaging for specific clinical situations. ${ }^{[5]}$ An Italian study assessing the appropriateness of CT and MRI found that $77.6 \%$ of CT scans and $78.9 \%$ of MRI scans were appropriate. ${ }^{[6]}$ Appropriateness was associated with a confirmation of the diagnostic hypothesis, only one examination performed during hospital stay, and the anatomical scan region, with the musculoskeletal system being the least appropriate. For CT scans, appropriateness was also associated with not using contrast agent. ${ }^{[6]}$

While some studies have been done in South Africa (SA), there remains a scarcity of literature specific to the appropriateness of requests for CT and MRI scans. ${ }^{[7]}$ Work in Western Cape Province, SA, demonstrated the need for regular review: $22 \%$ of referrals from a community health centre to secondary hospitals, of which $70 \%$ were for radiological imaging, were found to be unnecessary. ${ }^{[7]}$ Another SA study found reporting errors in up to $57.3 \%$ of requested scans, which related to a scanty clinical history in the request and increased workload periods ${ }^{[8]}$ More recently, authors at a large urban trauma centre reviewed the appropriateness of a pan CT scan for blunt abdominal trauma and found that it influenced clinical management 
in $77 \%$ of patients. ${ }^{[9]}$ A study done in 2012 at George Hospital in the Western Cape evaluated the appropriateness of CT scans, whether there was a discrepancy between the level of physician experience, and whether there were interdepartmental differences. ${ }^{[10]}$ The authors found that of 219 scans, $53.0 \%$ were abnormal. Overall, $6.4 \%$ of scans were considered inappropriate, while interns and registrars did not request inappropriate scans. The aim of this study was to review the appropriateness of CT and MRI scans done during a 6-year followup study at a single facility.

\section{Methods}

This was a retrospective descriptive study. The study setting was George Hospital, a regional referral hospital in the Garden Route district of the Western Cape. The hospital has 266 beds, with 8 general specialties, a 6-bed high-care unit, an emergency centre and 4 theatres. The hospital serves an outlying population of 620000 , and the emergency centre manages 140 patients a day. The radiology department has 1 radiologist, 1 medical officer, 10 radiographers and 1 nurse. One average, the department conducts 600 - 700 CT scans a month (hospital statistics - unpublished data, 2020). Afterhours consultant reporting is shared between off-site contracted radiologists, with 1 radiographer on site in the hospital, covering the wards, emergency centre and theatre. MRI scans are outsourced to a local private radiology practice. Comparing George Hospital with other hospitals, there is a significant discrepancy in the number of CT scans done per district. Between January and July 2019, George Hospital performed 4405 scans, Somerset Hospital 2 195, Karl Bremer Hospital 1 925, Worcester Hospital 1847 and Paarl Hospital 1746 (Provincial Department of Health - unpublished data, 2019).

All CT and MRI scans performed during October 2018 at George Hospital, as well as MRI scans outsourced to a private hospital, were analysed consecutively. The time period (October) was chosen to be the same as in the 2012 study. Data sources included: patient folders on the electronic content management system, i.e. Enterprise Content Management (ECM), Clinicom (for patient registration-specific statistics), Single Patient Viewer and National Health Laboratory Service (NHLS) for blood and histology results. Variables included demographics, indication for scan, clinical history, requesting physician level of experience (first-year intern, secondyear intern, community-service medical officer, medical officer, registrar, consultant), as well as the requesting clinical department.

Patient-specific information included age, whether treated as an inpatient, outpatient or during an emergency centre visit, and whether the request form specified the anatomical region for the study. Residence was noted as local (residing in George) or the area in the Garden Route from where the patient was referred. For each imaging report, the clinical history provided in the request was checked against ACR guidelines, and the following were noted: impact of the scan on the clinical course of the patient, whether the patient was referred to a specialty department, further investigations and if the patient was discharged.

Radiology department variables included whether the study was performed during normal working hours, i.e. between $08 \mathrm{~h} 00$ and 16h00 from Monday to Friday. After-hours scans were labelled as being performed after $16 \mathrm{~h} 00$ until $08 \mathrm{~h} 00$ on weekdays and from Friday $16 \mathrm{~h} 00$ throughout the weekend until $08 \mathrm{~h} 00$ on Monday. Timing was relevant, as there are fewer radiographers, support staff and senior doctors available after hours. The dose of radiation was calculated per milliampere seconds (mAs), dose-length product $(\mathrm{mGy}-\mathrm{cm})$ was noted for each patient, as well as whether contrast media were used.
All scans were classified as usually appropriate (UA), might be appropriate (MBA) or not appropriate (NA), according to ACR guidelines. ${ }^{[5]}$ The ACR continually updates and publishes appropriateness criteria, which are evidence based and annually reviewed by expert panels, including non-radiologists. The ACR supplies clinical guidelines to assist clinicians of various levels of experience in making initial decisions regarding radiological tests and interventions. ${ }^{[11]}$ Currently, there are 190 diagnostic imaging and interventional radiology topics, with 938 variants and $>1680$ clinical scenarios. The guidelines are accessible online free of charge and can be printed or stored after registration by email. Every CT and MRI request provided to the radiology department contains the patient's hospital number, name, location, referring doctor's name, specialty, brief clinical history and blood results, specifically if a contrasted study or intervention is necessary.

Every request was categorised as being UA, MBA or NA by searching the ACR database, using the requesting history. A score from 1 to 9 was assigned, 1 being least appropriate and 9 being most appropriate, corresponding to the three categories. The ACR evidence tables, literature searches, appendices, strength of evidence assessment and final rating tabulations for each recommendation are available (Appendix A: http://samj.org.za/public/sup/14860.pdf (an example of a specific clinical variant)). Where specific clinical scenarios were unclear, the ACR was contacted via email with the presenting history, and guidance was given regarding the topic category. This was only needed for 1 CT request.

Data were anonymised and captured on Microsoft Excel 2005 (Microsoft, USA). Stratified analysis was done using $\chi^{2}$ tests, comparing some of the data with the 2012 study results, with $p<0.05$ considered statistically significant. Simple statistics are described in terms of means and frequencies. Data quality was crossreferenced with hard copies in the radiology CT scan documentation book and cross-checked with the Xero and Picture Archiving and Communication System (PACS) (RamSoft, USA) electronic patient information systems exclusive to the radiology department.

Inclusion criteria comprised all patients undergoing a CT or MRI scan during October 2018, validated against manual record-keeping and PACS/Xero digital records. Participants were excluded if there were insufficient electronic data available to apply ACR criteria.

\section{Ethical approval}

Ethical approval for the study was obtained from the Higher Research Ethics Committee, Stellenbosch University (ref. no. N19/09/125), the Western Cape Department of Health (ref. no. WC_201912_013) and the Management and Ethics Committee, George Hospital.

\section{Results}

A total of 508 CT and 9 MRI scans were identified for analysis. Two CT studies were excluded, as they did not meet the inclusion criteria, bringing the total number to 515 . The mean age of the patients was 47 years, with the youngest patient being 1 year and the oldest 93 years (Fig. 1).

Of the studies performed, 419 (81.4\%) were found to be UA, $38(7.4 \%) \mathrm{MBA}$ and $58(11.2 \%)$ NA. While there were only 9 MRI scans, all were appropriately ordered (Fig. 2).

With regard to CT scans that qualified as UA $(n=410), 348(84.9 \%)$ needed further intervention and had a demonstrable impact on the clinical management of the patient, whereas $24(63.2 \%)$ of the MBA and $41(72.0 \%)$ of the NA scans could not demonstrate that these influenced the clinical management plan.

Most CT scans were requested by medical officers ( $n=255 ; 50.1 \%)$, followed by consultants $(n=117 ; 23.1 \%)$ and junior doctors (first- and 


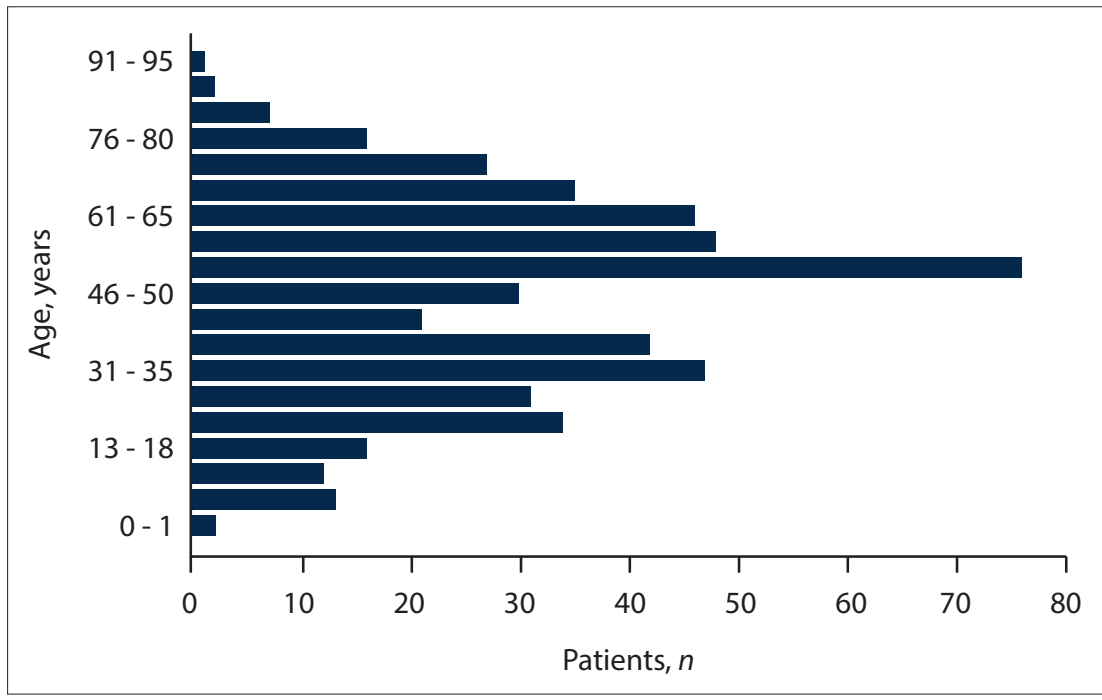

Fig. 1. Age distribution of patients.

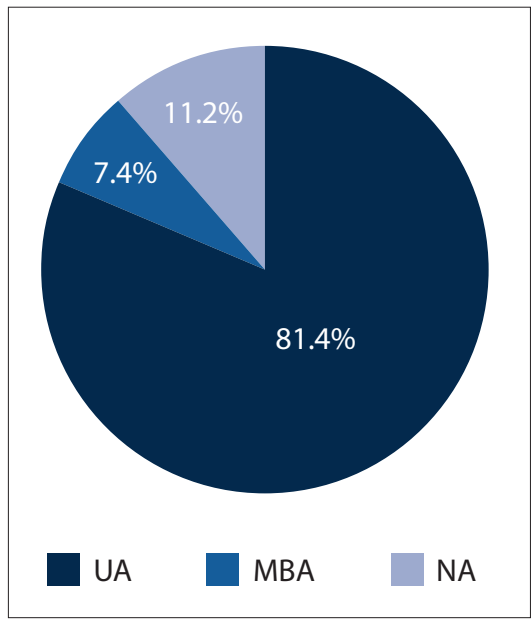

Fig. 2. Overall appropriateness score of all studies performed. (UA = usually appropriate; $M B A=$ might be appropriate; $N A=$ not appropriate.)

Table 1. Appropriateness of scans in relation to requesting practitioner seniority compared with 2012 study results

\begin{tabular}{lllllll}
\hline Practitioner & $\begin{array}{l}\text { Usually } \\
\text { appropriate, } \boldsymbol{n}(\%)\end{array}$ & $\begin{array}{l}\text { Might be } \\
\text { appropriate, } \boldsymbol{n}(\%)\end{array}$ & $\begin{array}{l}\text { Not } \\
\text { appropriate, } \boldsymbol{n}(\%)\end{array}$ & $\begin{array}{l}\text { Non-codable, } \boldsymbol{n}(\%) \\
\text { Total, }\end{array}$ & $\boldsymbol{N}(\%)$ & $\boldsymbol{p}$-value \\
\hline Consultant, Oct. 2012 & $71(63.4)$ & $13(11.6)$ & $6(5.4)$ & $22(19.6)$ & $112(100)$ & 0.00000 \\
Consultant, Oct. 2018 & $120(95.2)$ & $4(3.2)$ & $2(1.6)$ & $0(0)$ & $126(100)$ & \\
Registrar, Oct. 2012 & $6(66.7)$ & $3(33.3)$ & $0(0)$ & $0(0)$ & $9(100)$ & 0.44 \\
Registrar, Oct. 2018 & $19(86.4)$ & $3(13.6)$ & $0(0)$ & $0(0)$ & $22(100)$ & \\
Medical officer, Oct. 2012 & $54(65.8)$ & $15(18.3)$ & $4(4.8)$ & $9(11)$ & $82(100)$ & 0.000001 \\
Medical officer, Oct. 2018 & $222(74.7)$ & $26(8.8)$ & $49(16.5)$ & $0(0)$ & $297(100)$ & \\
Intern, Oct. 2012 & $6(66.7)$ & $3(33)$ & $0(0)$ & $0(0)$ & $9(100)$ & 0.037 \\
Intern, Oct. 2018 & $58(82.9)$ & $5(7.1)$ & $7(10)$ & $0(0)$ & $70(100)$ &
\end{tabular}

second-year interns and community-service medical officers) ( $n=112 ; 22.1 \%)$. Registrars were responsible for only $22(4.3 \%)$ of the requests. Medical officers requested most of the inappropriate scans $(n=40 ; 16.5 \%)$. Second-year interns requested the fewest number of scans $(n=6 ; 1.2 \%)$, as well as the lowest number of inappropriate scans, with registrars not requesting any inappropriate scans (Fig. 3).

Revisiting the results of the 2012 study, ${ }^{[6]}$ in 2018 consultants requested significantly more appropriate scans $(p<0.05)$, while medical officers requested significantly more inappropriate scans $(p<0.05)$ (Table 1$)$.

Of the CT and MRI scans, 392 (76.0\%) were completed during normal working hours and $123(24.0 \%)$ were done after hours. Of the after-hours scans, 37 of 123 (30.1\%) were NA and 10 (8.1\%) MBA. There were $76(61.8 \%)$ appropriate scans. In contrast, 21 of the 392 (5.4\%) in-hour scans were NA and 28 (7.1\%) MBA, giving the questionable in-hour scans as $n=49(12.5 \%)$ (Fig. 4).

The most frequently ordered CT requests was a scan of the brain $(n=270 ; 53.4 \%)$, followed by CT of the abdomen $(n=71 ; 14.0 \%)$ and CT of the chest $(n=70 ; 13.8 \%)$. Requests for orthopaedic scans accounted for 75 (14.8\%) of the workload. The brain CT requests were mostly for an uncontrasted scan. This requisition was also the most common inappropriate request (Fig. 5).

The departments of orthopaedics, paediatrics, psychiatry, family medicine and oncology used CT scans most appropriately, and the emergency department the least appropriately (Table 2 ).

Just fewer than half of the scans $(n=246$; $47.4 \%$ ) were performed on patients residing in the George area, with the rest being from surrounding hospitals. Scans were mostly performed on inpatients $(n=393 ; 76 \%)$, with the emergency medicine department requesting the most scans $(n=166 ; 32 \%)$, followed by internal medicine $(n=74 ; 14 \%)$ and psychiatry, with the lowest number of requests $(n=4 ; 0.8 \%)$. Only $40(7.7 \%)$ scans were direct referrals from surrounding district hospitals to the radiology department, without initial acceptance by emergency medicine or the appropriate specialty. After scanning was done, the patients were transferred to their respective hospitals for further management.
For the scans done in October, a total of 681440 mGy units and $4068358 \mathrm{mAs}$ were administered.

\section{Discussion}

Using ACR criteria, George Hospital used the CT and MRI suites appropriately for $81.4 \%$ of requests. This compares favourably with international studies. ${ }^{[6]}$ Comparing the findings with the 2012 study results in the same facility, these indicate a favourable trend, with an increase of $17.0 \%{ }^{[10]}$ In both studies, scans were ordered on a digital system, with a clinical history, level of requesting physician and existing laboratory results. The scans were performed as per standard operating procedure and reports evaluated retrospectively, with subsequent electronic notes for outcomes. There was a marked improvement in record-keeping and availability of data, as only 2 studies could not be included owing to lack of data availability compared with the 2012 study, where $32(0.12 \%)$ patients were excluded. ${ }^{[10]}$

Registrars were the most appropriate requesting physicians, with no inappropriate requests. Medical officers requested the most scans and also the highest number of 


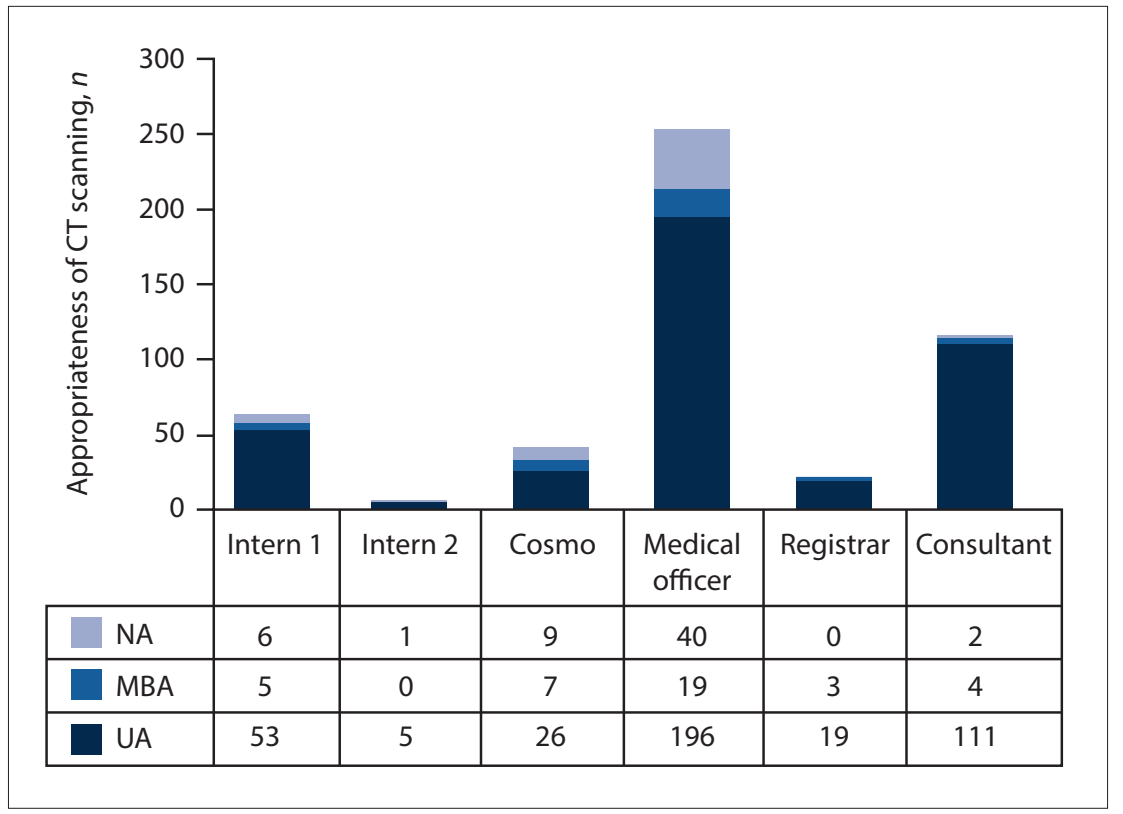

Fig. 3. Appropriateness of computed tomography scanning according to physician level of seniority. $(C T=$ computed tomography; $N A=$ not appropriate; $M B A=$ might be appropriate; $U A=$ usually appropriate; Cosmo = community-service medical officer.)

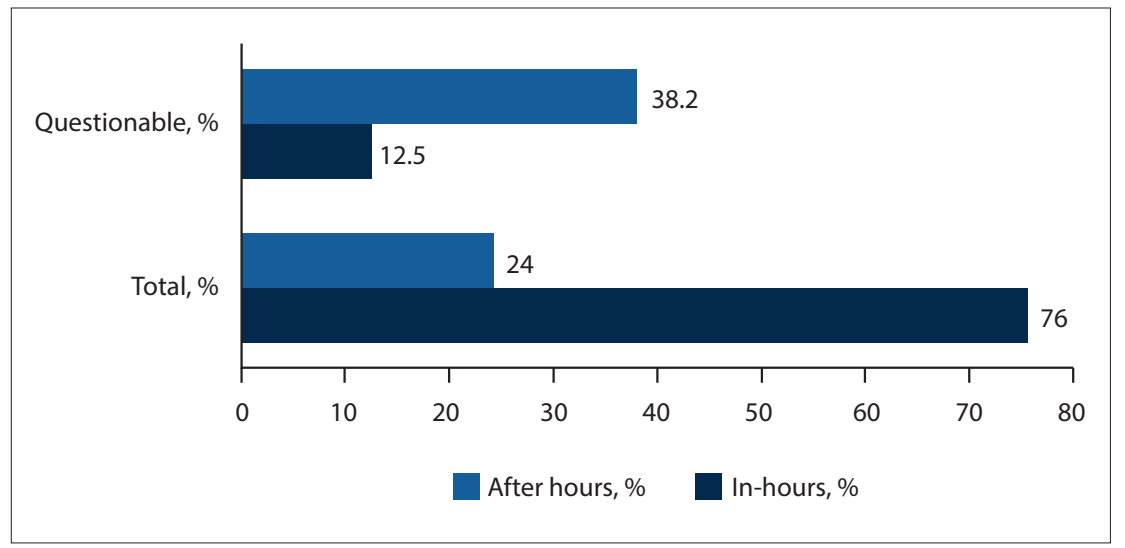

Fig. 4. Timing of scans and influence on the appropriateness score.

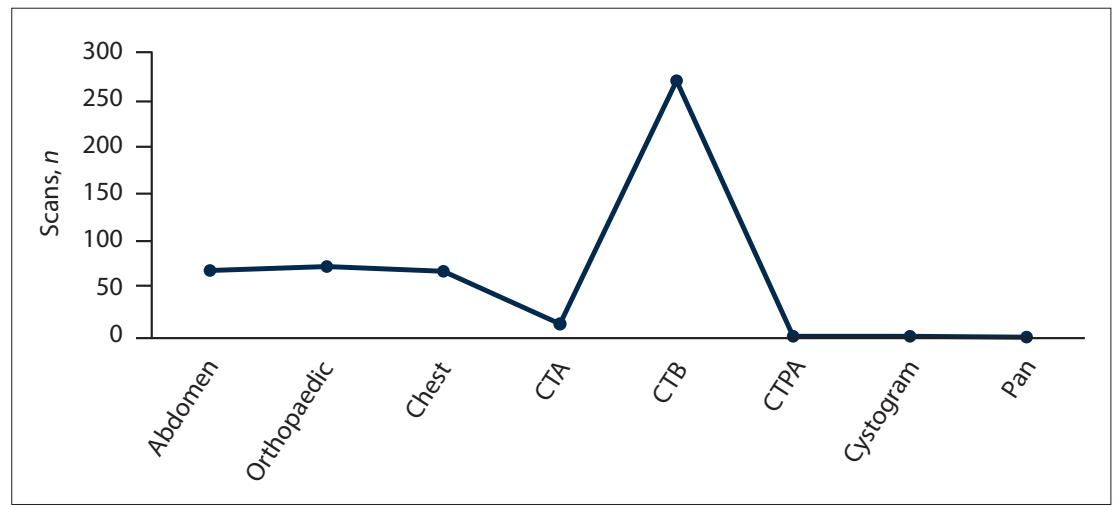

Fig. 5. Distribution of requested scans. $(C T A=$ computed tomography abdomen; $C T B=C T$ brain; $C T P A=C T$ pulmonary angiogram.)

inappropriate scans (16.5\%). Most of the inappropriate scans where done after hours. Although the bulk of the work was done during normal working hours, only $12.5 \%$ of such scans were questionable compared after hours. As medical officers are the professional category making most decisions after hours, this could explain some of these findings.

Requests were mostly for CT scans of the brain, which were also mostly inappropriately requested. As this occurred especially after hours, when there are fewer staff, by implication this added pressure on the radiology service, thus increasing potential error. This problem has been described before, where a direct correlation between increased workload and increased error rate in CT scan reporting was found. ${ }^{[8]}$ The most common error was missed findings in up to $57.3 \%$ of scans, reiterating the importance of an adequate clinical history provided to the radiologist. ${ }^{[8]}$ In our study, a poor or scanty clinical history was the main reason for inappropriateness. In some cases, the only clinical history provided was 'assault', with no additional mechanism, no Glasgow Coma Score and no mention of associated injuries. Other examples of scanty history included a CT scan of the brain for first-onset generalised tonic-clonic seizures while acutely intoxicated or with a positive urine drug screen. It is well known that the radiology report is influenced by the clinical history. ${ }^{[12,13]} \mathrm{A}$ more detailed clinical history would allow the radiologist to generate a more accurate report.

Other commonly found inappropriate CT scans were those done for abdominal polytrauma, where more readily available bedside investigations were not mentioned or used, e.g. e-FAST (extended focused assessment with sonography in trauma). Previous work concluded that $77 \%$ of pan CT scans for blunt polytrauma in a large SA trauma centre influenced management. ${ }^{[9]}$ The rest of the 'clinically negative scans' were clinically important because the clinical examination was unreliable. ${ }^{[9]}$ The concern is overutilising ionising radiation, where alternative investigative imaging could have been employed. However, some studies indicated a positive clinical impact in terms of decision-making from after-hours abdominal CT scans for acute pain, but the authors also concluded that a request with more clinical information was needed to enable more appropriate imaging. ${ }^{[14]}$

If international or a greater number of regional guidelines for performing CT scans were incorporated into the requesting database for easy reference, the appropriateness of requests and the accuracy and usefulness of reporting would improve. Some of these guidelines include the Canadian CT Rules, the NICE (National Institute for Health and Care Excellence) 
Table 2. Appropriateness of scans in relation to the requesting department

\begin{tabular}{lllll}
\hline Department & Scans, $\boldsymbol{n}$ & Usually appropriate & Might be appropriate & Not appropriate \\
\hline Emergency & 166 & 109 & 16 & 41 \\
Internal medicine & 74 & 68 & 3 & 3 \\
Surgery & 69 & 61 & 3 & 5 \\
Orthopaedics & 58 & 54 & 1 & 3 \\
Oncology & 32 & 29 & 3 & 0 \\
Ophthalmology & 29 & 26 & 2 & 1 \\
Family medicine & 30 & 27 & 2 & 1 \\
Paediatrics & 13 & 10 & 3 & 0 \\
Psychiatry & 4 & 4 & 0 & 2 \\
External referrals & 40 & 32 & 6 &
\end{tabular}

guidelines for CT scans, and the Western Cape Department of Health Guidelines. ${ }^{[15-19]}$ Incorporating evidence-based guidelines specific to the imaging study that was requested would alert the requesting physician to the possibility of an inappropriate scan and other modalities that would be more efficient and cost effective, e.g. ultrasonography.

All the MRI scans were appropriate. Possible reasons include a staggered approach with a limited number of available MRI slots per month, needing pre-approval by the medical manager. One reason for this gate-keeping is that MRI scanning is an outsourced service, with a significant cost implication. There was a marked increase in the use of ionising radiation since the 2012 study, with 258 more scans performed during the same calendar month - a $107 \%$ increase. The population growth in the Garden Route district for the same period was $\sim 4.6 \%{ }^{[20]}$ The number of MRI scans has more than halved from 27 to only 9 , but this is mainly owing to the abovementioned reasons. Regarding children, very few scans were ordered, and the paediatric department performed no inappropriate scans, indicating good practice regarding radiation exposure. This is a significant improvement since the 2012 study, when $18.7 \%$ of scans were inappropriately requested. It is evident that CT scans are preferred above MRI scans. One reason is that MRI scans are done in the private sector, necessitating a physician to accompany the child to the MRI facility for conscious sedation and monitoring. This routine practice limits the number of MRI scans being ordered.

When comparing the use of scans per sub-district, there was a clear discrepancy. Almost half of the scans were being performed in the George sub-district ( $n=246 ; 47.6 \%)$, followed by Mossel Bay sub-district ( $n=62 ; 12.0 \%)$ (53 km distance) and Knysna sub-district $(n=41 ; 8.2 \%)(60 \mathrm{~km}$ distance). Ease of access and availability have been shown to increase the number of paediatric CT scans performed, with an increased rate of negative scans. ${ }^{[21]}$ One explanation includes easy accessibility to the CT scanner at the regional hospital, resulting in disproportionately more scans being done.

\section{Recommendations}

- A pre-authorisation system where senior, experienced physicians grant approval for specialised investigations should be routine practice.

- More awareness of ACR criteria; the implications should be encouraged for all levels of requesting physicians. Implementing evidence-based guidelines as part of any requesting platform will improve reporting accuracy, efficiency and cost effectiveness. If fewer inappropriate scans are done, it will free up more time to report on appropriate scans.

- The ready availability and ease of ultrasound examination of the abdomen should be encouraged before using CT of the abdomen to minimise the use of ionising radiation. Increased training in ultrasound scanning will be necessary.
- Physicians from peripheral hospitals, who have the full history and have examined the patient, could reduce inappropriate scans and obtain a more accurate report by directly ordering and discussing CT scans with a senior doctor in a specific clinical department or directly with a radiologist.

\section{Study limitations}

This was a retrospective study, which always carries a risk of bias when interpreting data. The authors attempted to minimise this risk by using the same methods as in the previous study to improve the accuracy of the findings. Checking data between electronic and hard-copy data bases helped to minimise potential bias. It was not always clear whether junior doctors were ordering scans as part of their administrative duties in response to instructions from seniors, or whether they were doing so without such instructions. The usual practice would be to indicate, in the online ordering, the senior doctor who gave permission, but this information was occasionally omitted. While this study added new insights into clinical practice and governance to the local context and the topic in general, generalisability is limited, and more studies are needed to ascertain the appropriateness of CT and MRI scans in similar contexts in SA.

\section{Conclusions}

The aim of this study was to review the appropriateness of CT and MRI scans done in a 6-year follow-up study at a single facility. Eighty-one percent of scans were being ordered appropriately, which is an increase of $17 \%$ from the 2012 study. Scans that were most frequently requested inappropriately were CT scans of the brain. This was particularly true after hours. Pre-authorisation by experienced physicians and incorporation of guidelines would make requests more complete and possibly more appropriate. Future research, perhaps at other regional hospitals with similar contexts, would help to understand the appropriate use of this investigation better.

\section{Declaration. None.}

Acknowledgements. The authors thank the radiology department at George Hospital, specifically Dr M Kemp, for commitment to and support of the project. We also thank Ms T Esterhuizen of the Biostatistics Unit at Stellenbosch University for her support with the data analysis.

Author contributions. All authors conceptualised the research and contributed to various drafts of the manuscript. PEF collected and analysed the data and drafted the first manuscript. All authors approved the final manuscript.

Funding. None.

Conflicts of interest. None. 
1. Power SP, Moloney F, Twomey M, James K, O'Connor OJ, Maher MM. Computed tomography and patient risk: Facts, perceptions and uncertainties. World J Radiol 2016;8(12):902

2. CT scan market size, share, trends, growth, 2019 - 2024. https://www.marketdataforecast.com/marketreports/global-ct-scan-market (accessed 20 April 2020).

3. Schauer DA, Linton OW. National Council on Radiation Protection and Measurements report shows Schauer DA, Linton OW. National Council on Radiation Protection and Measurements report shows
substantial medical exposure increase. Radiology 2009;253(2):293-296. https://doi.org/10.1148/

4. Schmidt CW. CT scans: Balancing health risks and medical benefits. Environmental health perspectives. Nat Inst Environm Health Sci 2012;120:118-121. https://doi.org/10.1289/ehp.120-al18 5. American College of Radiology. ACR appropriateness criteria. https://www.acr.org/ClinicalResources/ACR-Appropriateness-Criteria (accessed 20 April 2020).

6. Bianco A, Zucco R, Lotito F, Pavia M. To what extent do hospitalised patients receive appropriate CT and MRI scans? Results of a cross-sectional study in southern Italy. BMJ Open 2018;8(2):e018125. https://doi.org/10.1136/bmjopen-2017-018125

7. Richards DB, Jacquet GA. Analysis of referral appropriateness in the Western Cape, South Africa, and implications for resource allocation. Afr J Emerg Med 2012;2(2):53-58.

8. Terreblanche OD, Andronikou S, Hlabangana LT, Brown T, Boshoff PE. Should registrars be reporting after-hours CT scans? A calculation of error rate and the influencing factors in South Africa. after-hours CT scans?

9. Oosthuizen G, Bruce JL, Bekker W, Shangase N, Laing GL, Clarke DL. Pan computed tomography for blunt polytrauma: Are we doing too many? S Afr Med J 2016;106(8):801-803. https://doi.org/10.7196 SAMJ.2016.v106i8.10376

10. Becker J, Jenkins LS, de Swardt M, Sayed R, Viljoen M. Appropriateness of computed tomography and magnetic resonance imaging scans in the Eden and Central Karoo districts of the Western Cape Province, South Africa. S Afr Med J 2014;104(11):762-765. https://doi.org/10.7196/SAMJ.8158

11. Wintermark M, Sanelli PC, Anzai Y, Tsiouris AJ, Whitlow CT. Imaging evidence and recommendation for traumatic brain injury: Conventional neuroimaging techniques. J Am Coll Radiol 2015;12(2).
12. Troude P, Dozol A, Soyer P, et al. Improvement of radiology requisition. Diagn Intervention Imaging 2014;95(1):69-75.

13. Khorasani R. Computerized physician order entry and decision support: Improving the quality of care. Radiographics 2001;21:1015-1018

14. Karia M, Seager M, Rafique A, Sheth H. The diagnostic utility and clinical impact of after-hours CT scans of the abdomen and pelvis investigating abdominal pain. Sci World J 2017:6. https://doi. org $/ 10.1155 / 2017 / 4028352$

15. Hassan Z, Smith M, Littlewood S, et al. Head injuries: A study evaluating the impact of the NICE head injury guidelines. Emerg Med J 2005;22(12):845-849.

16. Dunning J, Lecky F. The NICE guidelines in the real world: A practical perspective. Emerg Med J 2004:21(4):404-407.

17. Boyle A, Santarius L, Maimaris C. Evaluation of the impact of the Canadian CT head rule on British practice. Emerg Med J 2004;21:426-428.

18. Western Cape Department of Health. Head Injury Guidelines Handbook for the Western Cape. Cape Town: WCDoH, 2011:1-23.

19. Corley J, Barthèlemy EJ, Lepard J, et al. Comprehensive policy recommendations for head and spine injury care in low- and middle-income countries. World Neurosurg 2019;132:434-436.

0. Garden Route Intersectoral Development Plan. http://www.gardenroute.gov.za/documents/finalreviewed-idp-2018-2019/ (accessed 19 April 2020).

21. Arnold M, Moore SW. Paediatric blunt abdominal trauma. Are we doing too many computed tomography scans? S Afr J Surg 2013:51(1):26-31.

Accepted 3 August 2020 